\title{
Restoration of the coupling process and normalization of bone mass following successful treatment of endogenous Cushing's syndrome: A prospective, long-term study
}

\author{
Cybèle Kristo $^{1,2}$, Rune Jemtland ${ }^{1}$, Thor Ueland ${ }^{1,2}$, Kristin Godang ${ }^{1}$ and Jens Bollerslev ${ }^{1}$ \\ ${ }^{1}$ Section of Endocrinology and ${ }^{2}$ Research Institute of Internal Medicine, Department of Medicine, Rikshospitalet University Hospital, N-0027 Oslo, Norway \\ (Correspondence should be addressed to C Kristo; Email: cybele.kristo@rikshospitalet.no)
}

\begin{abstract}
Objective: Endogenous Cushing's syndrome (CS) is associated with bone loss and an increased risk of fractures. However, the long-term outcome of treatment on bone health has not been adequately clarified.

Design: We followed 33 patients with active CS prospectively before and twice after treatment (mean follow-up $33(n=25)$ and 71 months $(n=18)$, respectively). The patients were compared to age-, sex- and body mass index (BMI)-matched controls, also followed longitudinally.

Methods: Bone mineral indices (bone mineral density (BMD), bone mineral content (BMC) and bone area) were evaluated in the lumbar spine (LS), femoral neck (FN), and total body (TB) by dual-energy X-ray absorptiometry (DXA). Biochemical markers of bone turnover were assessed by serum levels of osteocalcin and C-terminal telopeptides of Type-1 collagen (CTX-1).

Results: Mann-Whitney rank sum tests showed that BMD of the LS, FN and TB was reduced by $14.8 \%$ $(P<0.001), 15.7 \%(P<0.001)$, and 9.2\% $(P<0.001)$ in CS vs. controls at baseline, with markedly reduced serum osteocalcin $(P=0.014)$ and increased CTX-1 $(P=0.012)$ levels, but no correlation between markers. At first follow-up, BMD was increased in LS $(7.9 \%, P<0.001)$ and FN $(3.5 \%$, $P=0.003)$ compared to baseline. The time-dependent rise in BMD (LS $(r=0.59 ; P=0.002)$ and FN $(r=0.52 ; P=0.007)$; Spearman's rank correlation), in CS was paralleled by increased osteocalcin $(275 \%, P<0.001)$ and correlation between biochemical markers $(r=0.92, P<0.001$; Pearson's correlation). TB BMD did not increase significantly before the second follow-up, when BMD Z-scores were normalized in all three compartments.

Conclusion: Our observations demonstrate restoration of coupled bone remodeling and normalization of bone mineral density in all measured skeletal compartments of treated CS patients after prolonged recovery, first significant in predominantly trabecular bone (i.e. lumbar spine).
\end{abstract}

European Journal of Endocrinology 154 109-118

\section{Introduction}

Glucocorticoid (GC)-induced bone loss is a frequent and serious complication in patients with endogenous Cushing's syndrome (CS) $(1,2-11)$ as well as in patients on GC therapy (12), leading to an increased risk of low-energy fractures $(8,13-15)$. While patients receiving GC therapy usually suffer from a primary disease that may have negative influence on bone mass by itself, CS (i.e. hypercortisolism, caused by a tumor in the pituitary gland or in the adrenal cortex) is not associated with an underlying primary condition known to affect bone metabolism directly. Thus, CS is considered to be a useful clinical model to investigate the 'pure' effects of excess GC on bone metabolism, minimizing other confounding factors. Previous reports have provided evidence that bone loss due to hypercortisolism (exogenous or endogenous) is most pronounced in areas with trabecular bone such as the lumbar spine (predominantly trabecular bone) and femoral neck (FN) (less trabecular bone, more cortical bone) (1-11, 16). Accordingly, fracture risk appears to be highest in the vertebrae and ribs of these patients $(8,13,14)$. In contrast, the effect of GC on skeletal sites with predominantly cortical bone is less well-documented $(5,9,16,17)$. Few reports have assessed restoration of bone mass after treatment of patients with active CS and there is a scarcity of long-term prospective studies $(1,3,11,18)$, reflecting the low incidence of CS $(1-10$ per million per year) (19). Most previous studies have described partial restoration of bone mass after treatment $(1,3,11)$. However, little is known about the long-term outcome of bone health in CS, because most studies have re-evaluated the patients after a short (3-6 months) or intermediate (12-24 months) time of follow-up $(3,6,11)$. Moreover, it has 
not been established whether bone mass at skeletal sites with predominantly trabecular or cortical bone is affected differently by correction of hypercortisolism after successful surgical treatment.

The exact mechanism by which GC influences bone metabolism is still not completely understood (12). While there is evidence that bone loss due to excess GC, at least in part, may be secondary to hypogonadism, decreased intestinal calcium absorption and renal calcium reabsorption, in addition to loss of muscle mass and strength, it is obvious that GCs have direct potent effects on bone cells. The skeletal effects of GC excess dominate over those of orchidectomy in mice, indicating that bone loss in this model occurs independently of changes in gonadal status (20). A characteristic feature of GC-induced bone loss is a depression of bone turnover, which is likely to be related to reduced bone formation (3, 4, 9, 16, 21-26), although bone resorption has also been shown to be increased in some studies (27). Accordingly, animal studies and in vitro experiments have shown that chronic GC administration decreases the birth rate and function of osteoblasts, increases apoptosis of mature osteoblasts and osteocytes $(28,29-32)$ and stimulates and prolongs osteoclast formation, activity and survival $(27,33,34)$.

The aim of the present study was to evaluate longterm effects of successful surgical treatment on bone densitometric parameters and bone turnover in patients with CS. Patients were evaluated longitudinally over an interval of mean 71 months, and were also compared to a closely matched control group, also followed longitudinally. Moreover, bone mass and projected bone area was evaluated in different compartments in order to assess whether GCs exert differential effects at skeletal sites with predominantly cortical or trabecular bone. Finally, we estimated alterations in biochemical markers of bone turnover, to explore possible mechanisms involved in the pathogenesis of GCinduced bone loss in CS patients with active disease and during the recovery phase after cure.

\section{Material and methods}

\section{Subjects}

CS patients with pituitary adenoma (Cushing's disease) or adrenal cortex adenoma diagnosed in our center between 1994 and 1999 were consecutively recruited to this study, after giving signed informed consent (5, 16). The study was approved by the local ethical committee and conducted according to the Declaration of Helsinki II.

All patients were diagnosed with CS based on a thorough clinical evaluation and biochemical work-up, as previously reported (5). Prior to surgical treatment, a baseline population of 33 CS patients ( 24 women, 9 men) was systematically evaluated and matched with
33 healthy controls. Of these, 25 patients (17 women, 8 men) were followed longitudinally for a mean of 33 months (range 5-69 months), and among these, 18 patients (13 women, 5 men) were further re-evaluated at a second follow-up, 71 months (range 43-108 months) after treatment. The controls, whom were matched for age, sex, and body mass index (BMI), were evaluated at baseline and then after a mean of 50 months (range 26-56 months). Prior to operative treatment, none of our patients received medication in order to block steroid synthesis. Patients with Cushing's disease were treated by transsphenoidal microsurgery; of these, four patients with relapse were subsequently subjected to bilateral adrenalectomy and were substituted with cortisone acetate $37.5 \mathrm{mg} /$ day and fludrocortisone $0.1 \mathrm{mg} /$ day. GC substitution was stopped for 24 hours before blood sampling in the patients on this medication. At baseline, none of the male patients had hypogonadism defined as a testosterone/SHBG (sex hormone binding globulin) ratio of less than $25 \%$ (mean ratio in the population 55\%, normal range $=25-200 \%$ ). Six women diagnosed with CS had regular menstruation, three had entered menopause (two were hysterectomized) and fifteen had amenorrhea for a mean of 27 months (range 6-60 months). None of the patients were substituted with sex hormones, thyroxine or growth hormone (GH). At the second follow-up, one of two men who had developed partial pituitary deficiency was substituted with testosterone and the other with GH. Five women had still regular menstruation, whereas two of eight women who had entered the menopause were substituted with hormone replacement therapy and two with dehydroepiandrosterone (DHEA).

\section{Methods}

All patients had clinical and biochemical findings of CS at baseline. The diagnosis was confirmed by an increase in daily urinary cortisol excretion, increase in basal serum cortisol concentrations with lack of physiological diurnal rhythm, lack of urinary and serum cortisol suppression after 2-day dexamethasone suppression test (DST, $0.5 \mathrm{mg} \times 4$ per day, sampling $2 \mathrm{~h}$ after the last tablet), and a low-dose DST (1.0 or $1.5 \mathrm{mg}$ depending on weight at follow-up) according to international standards (35). For patients with Cushing's disease the diagnosis was confirmed by inappropriately high plasma adrenocorticotropin (ACTH) concentrations and magnetic resonance imaging (MRI) of pituitary gland (eventually followed by sinus petrosus sampling), and for patients with adrenal cortex adenoma, suppressed plasma ACTH and computed tomography (CT) scanning of the adrenal glands.

Following treatment (first and second follow-up), all patients presented with normalized diurnal variation of serum cortisol, in addition to levels of 24-h urine free cortisol within the normal range. DST was considered normal when serum cortisol was suppressed 
to less than $40 \mathrm{nmol} / \mathrm{l}$. For ten patients at first follow-up and six patients at second follow-up, serum cortisol was $>40 \mathrm{nmol} / \mathrm{l}$ at DST. However, none of these patients showed differences in biochemical parameters or bone mass indices (bone mineral density (BMD), bone mineral content (BMC) and area), as compared to patients with DST $<40 \mathrm{nmol} / \mathrm{l}$, nor did they present with any clinical signs of relapse. All patients had normal liver and kidney function, as judged by serum levels of ASAT (aspartat aminotransferase), ALAT (alanin aminotransferase), albumin and creatinine.

\section{Osteodensitometric measurements}

$\operatorname{BMD}\left(\mathrm{g} / \mathrm{cm}^{2}\right)$, BMC $(\mathrm{g})$ and projected bone area $\left(\mathrm{cm}^{2}\right)$ were measured in the LS (L2-L4, anterior-posterior projection), the left FN and total body (TB), using DXA (Dual-Energy X-ray Absorptiometry; Lunar DPX-L, software version 4.6c, Lunar Corporation, Madison, WI) (36). BMD Z-scores are given with reference to normative data provided by the manufacturer. The precision error of LS was about 1\%, FN $1.5 \%$ and TB $0.5 \%$, independent of the operator (16).

\section{Blood sampling}

Blood samples were drawn between $0800 \mathrm{~h}$ and $1000 \mathrm{~h}$, after overnight fasting. For collection of serum, blood was drawn into pyrogen-free tubes (Becton Dickinson, San Jose, CA) without additives. The tubes were immediately immersed in ice water, allowed to clot for 2 hours, and centrifuged at $1000 \mathrm{~g}$ at $4 \mathrm{C}$ for 10 minutes; serum was stored at $-80^{\circ} \mathrm{C}$ until analyzed. All samples were thawed three times, and samples from a given individual were run in the same assay to minimize run-to-run variability.

\section{Biochemical markers of bone turn-over}

Osteocalcin was measured by immunoradiometric assay (IRMA) with a commercial kit (Incstar Corporation,
Stillwater, MI), that measures intact osteocalcin 1-49 . Degradation products of the C-terminal telopeptides of Type-I collagen (CTX-1) were measured with an enzyme immunoassay (EIA) (Crosslaps; Osteometer BioTech A/S, Herlev, Denmark). Serum and free cortisol in 24-h urine was measured by radioimmunoassay (RIA), using commercial kits from Orion Diagnostica, (Espoo, Finland). Cortisol and dehydroepiandrosterone levels were analysed using standard laboratory methods. The intra- and inter-assay coefficients of variation were $<7 \%$ for all assays.

\section{Statistical analysis}

Statistical analyses were performed by SPSS for Windows (version 12.0; SPSS Inc., Chicago, IL). Wilcoxon signed rank sum test was used to analyze changes in bone mass at different observation times. Mann-Whitney Rank-Sum test was used to compare variables of patients and controls. Relationships between two variables in BMD, BMC and bone area were tested using Spearman's rank correlation test and Pearson's correlation test for bone turnover markers. $P<0.05$ was considered statistically significant.

\section{Results}

The clinical characteristics and biochemical findings of the study populations at baseline and at regular intervals throughout the observation period are given in Table 1 .

\section{Time-dependent changes in bone mass indices at multiple skeletal sites before and after successful treatment of $\mathrm{CS}$}

$\mathrm{BMD}, \mathrm{BMC}$ and bone area were determined in CS patients by DXA at baseline and at two consecutive follow-ups (means 33 and 71 months respectively)

Table 1 Clinical characteristics and laboratory data before and after surgical treatment of CS patients, and during longitudinal follow-up in controls.

\begin{tabular}{|c|c|c|c|c|c|}
\hline & \multicolumn{3}{|c|}{ CS } & \multicolumn{2}{|c|}{ Controls } \\
\hline & $\begin{array}{l}\text { Baseline } \\
(n=33)\end{array}$ & $\begin{array}{l}\text { First follow-up } \\
\quad(n=25)\end{array}$ & $\begin{array}{l}\text { Second follow-up } \\
(n=18)\end{array}$ & $\begin{array}{l}\text { Baseline } \\
(n=33)\end{array}$ & $\begin{array}{c}\text { Follow up } \\
(n=25)\end{array}$ \\
\hline Gender (Female/male) & $33(24 / 9)$ & $25(17 / 8)$ & $18(13 / 5)$ & $33(24 / 9)$ & $25(17 / 8)$ \\
\hline Age (years) & $43 \pm 2$ & $47 \pm 2$ & $50 \pm 3$ & $43 \pm 2$ & $47 \pm 2$ \\
\hline Diagnosis (pituitary/adrenal) & $26 / 7$ & $20 / 5$ & $14 / 4$ & & \\
\hline BMI $\mathrm{kg} / \mathrm{m}^{2}$ & $29.0 \pm 1$ & $27.3 \pm 1$ & $26.9 \pm 1$ & $27.6 \pm 1$ & $27.5 \pm 1$ \\
\hline Body weight (kg) & $82.6 \pm 2.5$ & $78 \pm 3$ & $75.6 \pm 4$ & $81.2 \pm 2.4$ & $83.8 \pm 2.9$ \\
\hline Serum cortisol $(\mathrm{nmol} / \mathrm{l}) 0800 \mathrm{~h}$ & $627 \pm 42^{* \star}$ & $259 \pm 28 \dagger$ & $422 \pm 47$ & $409 \pm 33$ & $393 \pm 361$ \\
\hline Urinary cortisol (nmol/24h) & $1446 \pm 345$ & $193 \pm 21$ & $179 \pm 15$ & & \\
\hline DHEAS ( $\mu \mathrm{g} / \mathrm{dl})$ & $298 \pm 58^{*}$ & $60 \pm 11$ & & $142 \pm 13$ & \\
\hline Osteocalcin ( $\mu \mathrm{g} / \mathrm{L})$ & $3.1 \pm 0.3^{*}$ & $13.5 \pm 1.6 \dagger \dagger$ & $11.0 \pm 1.1$ & $4.26 \pm 0.4$ & $6.97 \pm 0.4$ \\
\hline CTX-1 (Crosslaps) (ng/ml) & $0.53 \pm 0.04^{*}$ & $0.75 \pm 0.13^{\text {ns }}$ & $0.73 \pm 0.08$ & $0.39 \pm 0.03$ & $0.58 \pm 0.06$ \\
\hline
\end{tabular}

Data are presented as mean \pm S.E.M. DHEAS, dehydroepiandrosterone. ${ }^{\star} P<0.05$, ${ }^{\star \star} P<0.001$, CS patients vs. controls at baseline. ${ }^{\dagger} P<0.05$,

${ }^{\dagger \dagger} P<0.001$, CS patients vs. controls at first follow-up. ns, not significant. 
after surgical treatment, as shown in Fig. 1 and Table 2. At baseline, bone mass (BMD and BMC) was reduced in all compartments of CS patients relative to controls, as was the TB projected area $(13 \%, P=0.01)$ (Table 2$)$. Already at the first follow-up (Fig. 1), highly significant improvements were demonstrated at the LS (BMD, 7.9\% $(P<0.001)$; BMC, $17.5 \%(P=0.001)$; area, $3.1 \%$ $(P=0.022))$ and $\mathrm{FN}(\mathrm{BMD}, 3.5 \%(P=0.003)$; BMC, $5.0 \%(P=0.025)$; area, $-4.5 \%(\mathrm{~ns}))$ relative to pretreatment values. Further gain in bone mass indices were observed at the second follow-up (relative to the first follow-up) at the LS (BMD, 10.7\% $(P=0.039)$; BMC, 7.8\% $(P=0.001)$; area, 6.9\% $(P=0.003))$ and FN (BMD, 4.6\% $(P=0.004)$; BMC, 3.6\% $(P=0.001)$; area, $-0.2 \%(\mathrm{~ns}))$. Whereas a trend towards increased BMC (12.6\%, (ns)) and bone area $(11.8 \%$, (ns)) was observed for $\mathrm{TB}$ at first follow-up (relative to baseline), BMD was unaltered $(0.9 \%,(P=0.16))$. By the second follow-up, a highly significant increase was observed in TB BMD $(5.1 \%,(P<0.001))$, with unchanged or only modest alterations in BMC $(0.8 \%$, (ns)) and bone area $(-1.8 \%$, (ns)). Thus, increases in BMD were observed at all measured skeletal sites in patients after treatment, however the changes $(P<0.05)$ appeared earlier at the LS and FN compared to TB, where the improvements reached statistical significance at the second follow-up.
A highly significant positive correlation between the changes in BMD and time after surgical treatment (mean 33 months) was demonstrated in the LS $(r=0.59 ; P=0.002)$ and $\mathrm{FN}(r=0.52 ; P=0.007)$, but not in TB $(r=0.27 ; P=0.30)$ (Fig. 2).

At baseline there was no correlation between serum cortisol $0800 \mathrm{~h}$ and osteodensitometric measurements. At the first follow-up we found a negative correlation between serum cortisol $0800 \mathrm{~h}$ and BMC at TB $(r=-0.48 ; \quad P=0.017)$, TB projected bone area $(r=-0.47 ; \quad P=0.021)$ and serum osteocalcin $(r=-0.41 ; P=0.04)$. Moreover, there was a negative correlation between serum cortisol and LS BMD at the second follow up $(r=-0.50 ; P=0.03)$ (data not shown).

\section{Normalization of bone mineral density at multiple skeletal sites in patients with CS after prolonged recovery}

Data are presented as Z-scores (25, 75 percentiles) BMD Z-scores for the various bone compartments in patients and healthy controls are given in Fig. 3. Patients with active CS had reduced BMD Z-scores at all measured sites (LS, - $1.50 \quad(-2.49,-0.76) ; \quad \mathrm{FN}, \quad-1.23$ $(-1.95,-0.52)$; and TB, $-1.37(-2.00,-0.71))$ at baseline. By comparison, BMD Z-scores for the controls
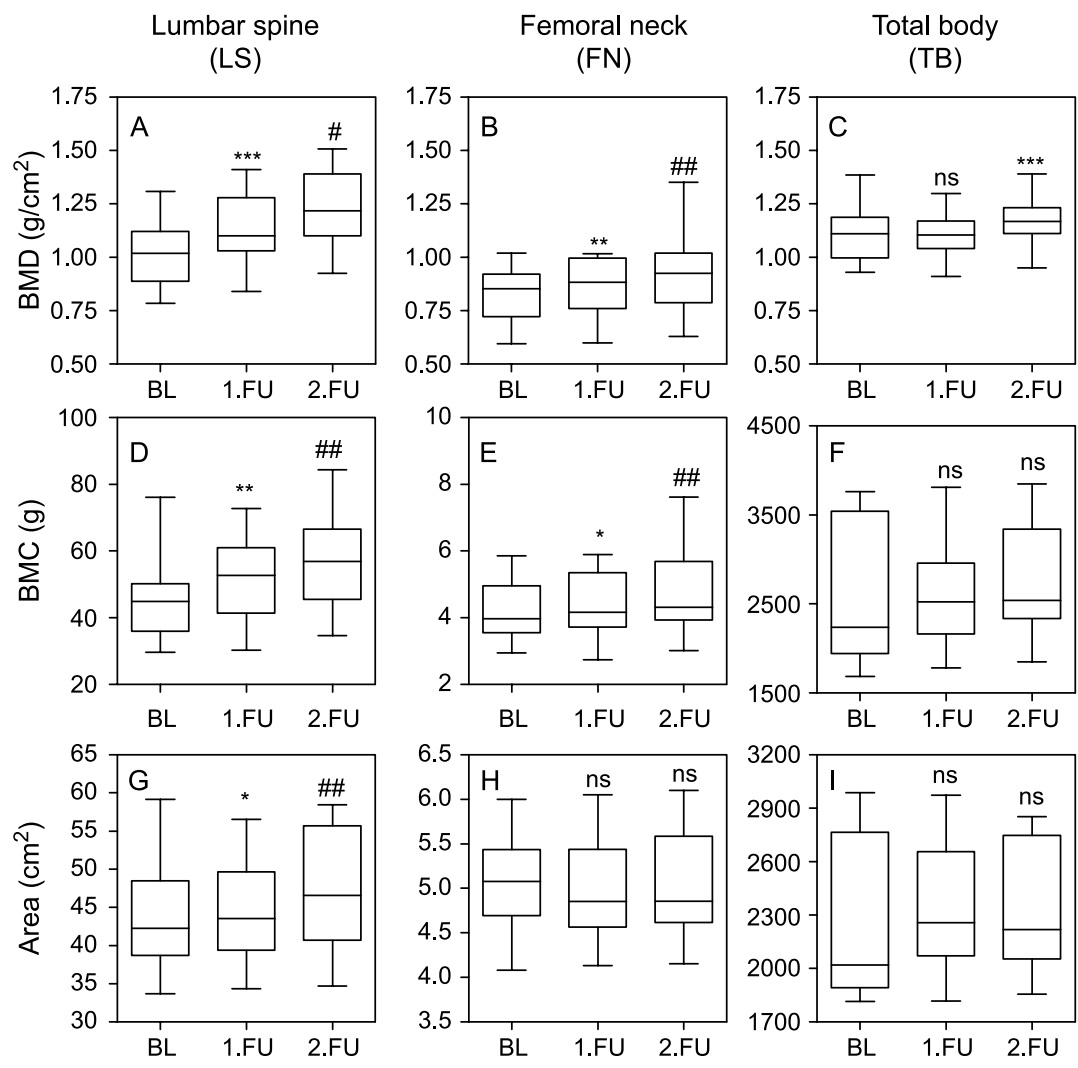

Figure $1 \mathrm{BMD}(\mathrm{A}-\mathrm{C})$, BMC (D-F) and projected bone area (G-I) before treatment and during longitudinal follow-up (mean 33 and 71 months, respectively) after surgical treatment of patients with CS. BL, baseline; 1.FU, first follow-up; 2.FU, second follow-up. Data are given as median and 25 and 75 percentiles. ${ }^{\star} P<0.05,{ }^{* \star} P<0.01$, ${ }^{* \star \star} P<0.001$; first follow-up vs. baseline. ${ }^{\#} P<0.05$, ${ }^{\# \#} P<0.01,{ }^{\# \# \#} P<0.001$; second $v s$. first follow-up. 
Table 2 Osteodensitometric parameters at various skeletal sites (see methods) before and after surgical treatment in CS patients and age-, sex-, and BMI-matched controls.

\begin{tabular}{|c|c|c|c|c|c|c|}
\hline \multirow[b]{2}{*}{ Osteodensitometry } & \multicolumn{3}{|c|}{ Cushing's } & \multicolumn{2}{|c|}{ Controls } & \multirow[b]{2}{*}{ CS vs. controls } \\
\hline & Baseline $(n=33)$ & First follow-up $(n=25)$ & Second follow-up $(n=18)$ & Baseline $(n=33)$ & Follow-up $(n=25)$ & \\
\hline LS BMD (g/cm²) & $1.05(0.94,1.13)$ & $1.15(1.03,1.26)$ & $1.22(1.11,1.39)$ & $1.23(1.09,1.33)$ & $1.20(1.06,1.35)$ & $\begin{array}{l}P<0.001(\mathrm{BL}) \\
P=0.149(\mathrm{FU})\end{array}$ \\
\hline LS Z-score (S.D.) & $-1.50(-2.49,-0.76)$ & $-0.50(-1.45,0.25)$ & $0.30(-0.80,1.33)$ & $-0.03(-0.934,0.87)$ & $-0.10(-0.95,1.15)$ & $\begin{array}{l}P<0.001(\mathrm{BL}) \\
P=0.159 \text { (FU) }\end{array}$ \\
\hline LS BMC (g) & $45.73(37.81,53.72)$ & $53.09(41.88,62.81)$ & $56.87(46.44,66.10)$ & $59.46(48.61,66.52)$ & $60.65(46.61,68.190$ & $\begin{array}{l}P=0.001(\mathrm{BL}) \\
P=0.051(\mathrm{FU})\end{array}$ \\
\hline LS $\left(\right.$ area $\left(\mathrm{cm}^{2}\right)$ & $42.42(39.44,50.25)$ & $43.83(41.66,52.47)$ & $46.58(41.21,55.52)$ & $46.15(41.19,52.39)$ & $46.36(41.33,53.13)$ & $\begin{array}{l}\mathrm{P}=0.17(\mathrm{BL}) \\
\mathrm{P}=0.135 \text { (FU) }\end{array}$ \\
\hline FN BMD $\left(\mathrm{g} / \mathrm{cm}^{2}\right)$ & $0.86(0.74 .0 .91)$ & $0.88(0.76,0.96)$ & $0.92(0.80,1.00)$ & $0.95(0.88,1.11)$ & $1.00(0.90,1.09)$ & $\begin{array}{l}P<0.001(\mathrm{BL}) \\
P=0.001(\mathrm{FU})\end{array}$ \\
\hline FN Z-score (S.D.) & $-1.23(-1.95,-0.52)$ & $-0.70(-1.40,0.05)$ & $0.10(-0.85,0.63)$ & $0.28(-0.76,0.67)$ & $0.10(-0.95,0.70)$ & $\begin{array}{l}P<0.001(\mathrm{BL}) \\
P=0.008(\mathrm{FU})\end{array}$ \\
\hline FN BMC (g) & $4.27(3.83,4.65)$ & $4.20(3.84,4.89)$ & $4.32(3.94,5.69)$ & $5.26(4.46,5.7)$ & $5.10(4.66,5.50)$ & $\begin{array}{l}P<0.001(\mathrm{BL}) \\
P=0.001(\mathrm{FU})\end{array}$ \\
\hline FN area $\left(\mathrm{cm}^{2}\right)$ & $5.12(4.76,5.49)$ & $4.97(4.58,5.44)$ & $4.86(4.63,5.56)$ & $5.9(4.88,5.99)$ & $5.07(4.37,5.62)$ & $\begin{array}{l}P<0.462(\mathrm{BL}) \\
P=0.613(\mathrm{FU})\end{array}$ \\
\hline TB BMD $\left(\mathrm{g} / \mathrm{cm}^{2}\right)$ & $\begin{array}{c}1.10(1.01,1.13) \\
n=23\end{array}$ & $\begin{array}{c}1.09(1.04,1.15) \\
n=16\end{array}$ & $\begin{array}{c}1.17(1.12,1.22) \\
n=11\end{array}$ & $\begin{array}{c}1.19(1.13,1.28) \\
n=23\end{array}$ & $\begin{array}{c}1.19(1.14,1.26) \\
n=16\end{array}$ & $\begin{array}{l}P<0.001(\mathrm{BL}) \\
P<0.001(\mathrm{FU})\end{array}$ \\
\hline TB Z-score (S.D.) & $\begin{array}{c}-1.37(-2.00,-0.71) \\
n=23\end{array}$ & $\begin{array}{c}-0.70(-1.55,0.05) \\
n=16\end{array}$ & $\begin{array}{c}0.45(-0.60,1.13) \\
n=11\end{array}$ & $\begin{array}{c}0.22(-0.35,0.89) \\
n=23\end{array}$ & $\begin{array}{c}0.40(-0.35,1.20) \\
n=16\end{array}$ & $\begin{array}{l}P<0.001(\mathrm{BL}) \\
P=0.002(\mathrm{FU})\end{array}$ \\
\hline TB BMC (g) & $\begin{array}{c}2348(2035,2635) \\
n=23\end{array}$ & $\begin{array}{c}2511(2197,2798) \\
n=16\end{array}$ & $\begin{array}{c}2544(2354,3293) \\
n=11\end{array}$ & $\begin{array}{c}2828(2557,3366) \\
n=23\end{array}$ & $\begin{array}{c}2783(2491,3181) \\
n=16\end{array}$ & $\begin{array}{l}P=0.001(\mathrm{BL}) \\
P=0.006(\mathrm{FU})\end{array}$ \\
\hline TB area $\left(\mathrm{cm}^{2}\right)$ & $\begin{array}{c}2091(1892,2422) \\
n=23\end{array}$ & $\begin{array}{c}2260(2094,2499) \\
n=16\end{array}$ & $\begin{array}{c}2218(2072,2734) \\
n=11\end{array}$ & $\begin{array}{c}2396(2223,2693) \\
n=23\end{array}$ & $\begin{array}{c}2387(2160,2601) \\
n=16\end{array}$ & $\begin{array}{l}P=0.010(\mathrm{BL}) \\
P=0.062(\mathrm{FU})\end{array}$ \\
\hline \multicolumn{7}{|c|}{$\begin{array}{l}\text { Patients were evaluated at baseline and at two successive follow-ups, mean } 33 \text { and } 71 \text { months after surgical treatment respectively. Controls were also followed longitudinally and evaluated at baseline and } \\
\text { follow-up, mean } 50 \text { months after inclusion. Data are given as median and } 25 \text { and } 75 \text { percentiles. Cross-sectional data: differences between CS patients and controls at baseline (BL) and first follow-up (FU), } \\
\text { respectively, are presented as } P \text { values in the last column. }\end{array}$} \\
\hline
\end{tabular}



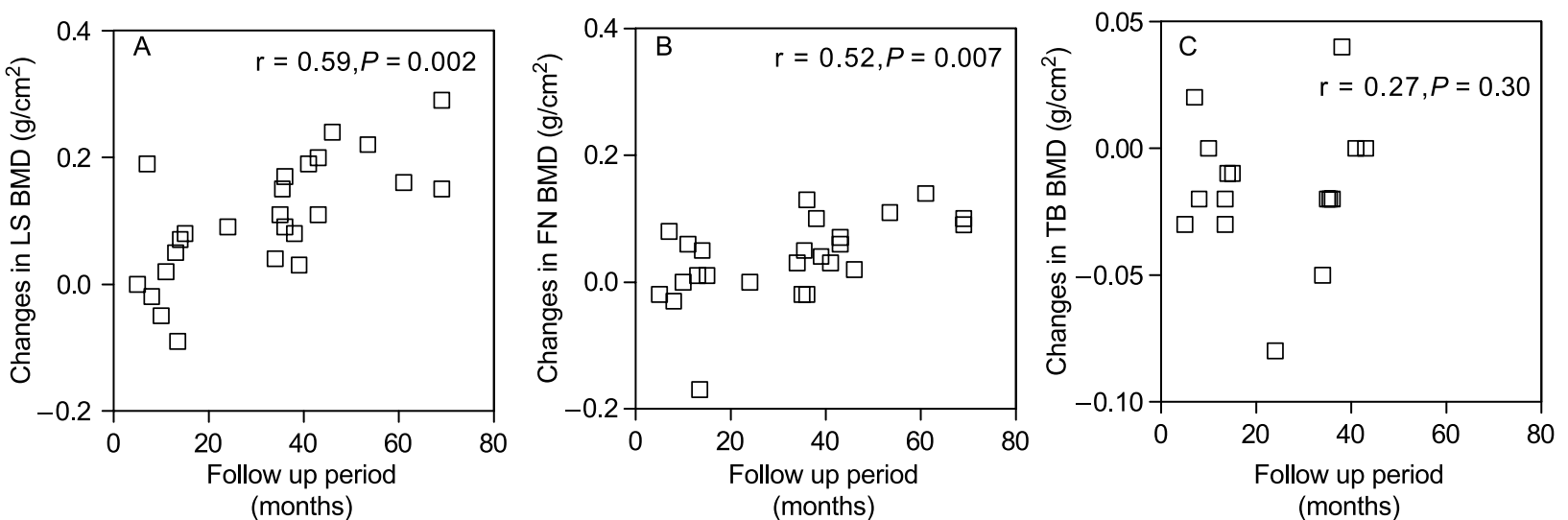

Figure 2 Correlations ( $r$ ) between changes in BMD in (A) LS, (B) FN and (C) TB, and recovery time (mean 33 months) after surgical treatment in patients with CS. Data are given as individual observations.

were not significantly different from the normative data of the reference population throughout the study. Following treatment, BMD Z-scores for the patients increased at the LS $(-0.50 \quad(-1.45,0.25)$, $P<0.001)$ and FN $(-0.70 \quad(-1.40,0.05)$, $P<0.001$ ) during the first follow-up (relative to baseline), with a trend towards an improvement also in TB $-0.70(-1.55,0.05), P=0.083)$. Thus, at the first follow-up there were no significant $(P=0.16)$ differences between LS BMD Z-score values in CS patients and controls, indicating normalization of bone mass at this site, whereas Z-scores were still significantly lower at FN $(P=0.010)$ and TB $(P=0.001)$. At the second follow-up, we observed a further rise in BMD Z-score at the LS $(0.30(-0.80,1.33), P<0.001)$, FN $(0.10(-0.85,0.63), P<0.001)$, and TB $(0.45$ $(-0.60,1.13), P<0.001)$, indicating normalization of BMD in all measured skeletal compartments with time.

\section{Biochemical markers of bone turnover: restoration of the bone remodeling process after successful treatment of CS}

To determine the rate of bone turnover, we also measured serum levels of osteocalcin (OC), a marker for bone formation, and cross-laps (CTX-1), a marker for bone resorption, both during longitudinal followup in CS patients (Fig. 4), and in relation to matched controls (Table 1). Compared to controls at baseline, serum OC levels were reduced by $26.9 \%(P=0.014)$ in the patients, whereas their serum CTX-1 levels were increased by $35.9 \%(P=0.012)$. At follow-up, levels of OC were markedly elevated (approx. 2-fold) in CS patients compared to controls $(P<0.001)$, whereas CTX-1 levels were only moderately enhanced (29.1\%, $P=0.68$ ). The longitudinal data (Fig. 4) show that serum osteocalcin levels increased markedly in the patients $(275 \%, P<0.001)$ at the first follow-up compared to baseline, and were sustained at high levels throughout the observation period. However, serum levels of CTX-1 tended to increase only modestly during the same time period. Notably, while no correlation was found between levels of bone formation and resorption markers before treatment in the patients $(r=0.32, \quad P=0.19)$, serum levels of osteocalcin and CTX-1 were significantly correlated both at first $(r=0.92, \quad P<0.001)$ and second follow-up $(r=0.90, P<0.001)$ after treatment, indicating that coupling between formation and resorption had been restored.

\section{Discussion}

The present study, which contains both longitudinal and cross-sectional data is, to our knowledge, the first to demonstrate normalization of bone mineral density in multiple skeletal compartments of patients with endogenous Cushing's syndrome after prolonged recovery time (mean 71 months). Whereas biochemical markers of bone turnover indicated uncoupled remodeling in the state of active disease, coupling seemed to be restored following treatment, leading to a marked increase in bone mass first appreciated in compartments rich in the metabolically active trabecular bone as in the LS, and most recently in the TB skeleton constituting of mainly $(80 \%)$ cortical bone.

Our baseline data demonstrate that patients with active CS displayed marked deficits in bone mass not only at the spine and $\mathrm{FN}$, as generally appreciated $(3-7,9,11,16,23)$, but also in the TB skeleton. The most pronounced bone loss was observed at the LS, consistent with the view that trabecular bone is a principal target for the detrimental effects of excess GC in the skeleton (12). Our longitudinal data demonstrating improvement in bone mass following treatment of CS extend previous findings by showing that the gain in LS BMD and FN BMD was positively related to follow-up time ( $r=0.59$ and $r=0.52$, respectively). 

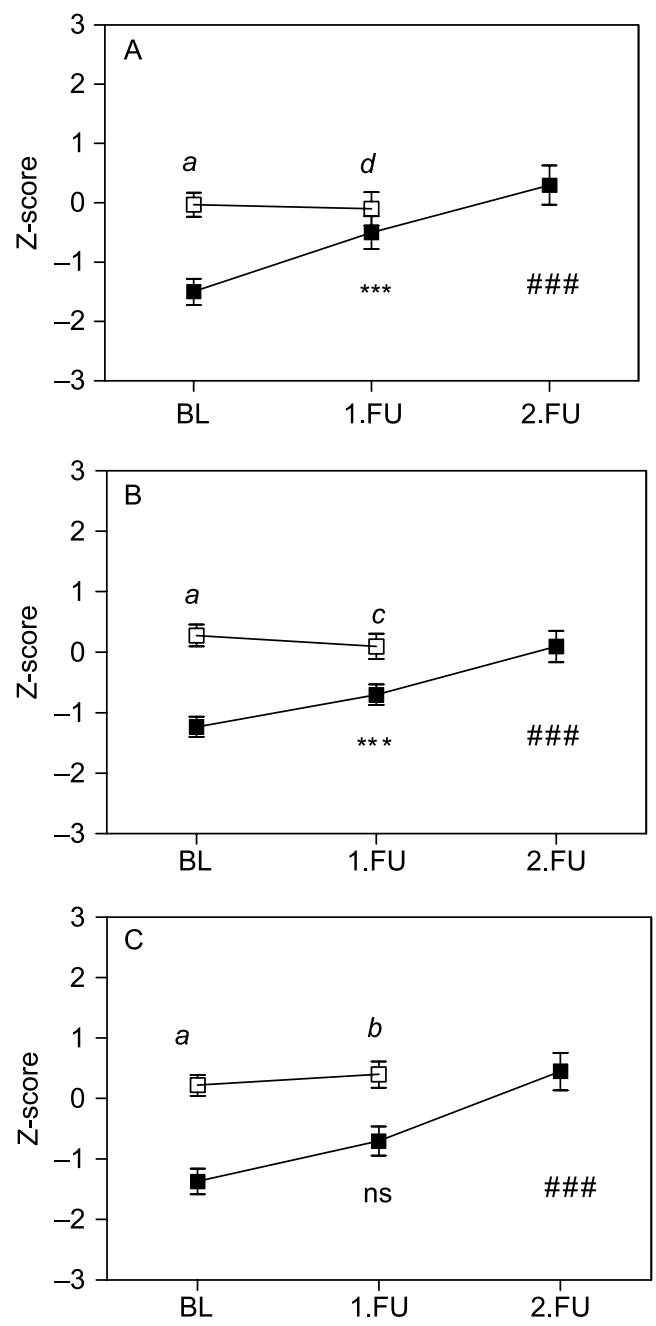

Figure 3 Z-score BMD values for (A) LS, (B) FN and (C) TB before (i.e. baseline) and after longitudinal follow-up in patients with CS ( $\square$ ), and in age-, sex-, and BMI-matched controls ( $\square$ ) also evaluated longitudinally. Data are presented as median \pm S.E.M. ${ }^{*} P<0.05,{ }^{* *} P<0.01,{ }^{* * *} P<0.001$; first follow-up vs. baseline in CS. ${ }^{\#} P<0.05$, ${ }^{\#} P<0.01$, ${ }^{\# \# \# ~} P<0.001$; second vs. first follow-up in patients with CS (see Table 2). ${ }^{a} P<0.001,{ }^{\mathrm{b}} P=0.001$, ${ }^{\mathrm{c}} P<0.05,{ }^{\mathrm{d}} P=\mathrm{ns}$; CS patients vs. controls.

This suggests that bone loss in CS is reversible after successful treatment, and is supported by normalization of BMD Z-scores in both sexes at all measured sites in CS patients with prolonged recovery (mean 71 months). This occurred despite the expected physiological agedependent bone loss, which was relatively modest in the study population (Fig.3) consisting mainly of premenopausal women. However, the time for significant measurable improvement in bone mass differed between the various skeletal compartments, and became significant first in the LS (mainly trabecular bone), then in the FN (less trabecular bone, more cortical bone), and lastly in TB (mainly cortical bone), possibly reflecting that a higher remodeling rate at the spine may have caused an earlier bone recovery than at cortical sites in the appendicular skeleton.

Interestingly, TB projected area was reduced compared to controls at baseline. In the follow-up period bone area increased significantly in the LS (and a similar trend in TB) throughout the observation period most likely reflecting periosteal apposition of new bone, in accordance with the persistent increase in serum levels of the bone formation marker osteocalcin. Decreased bone area has previously been demonstrated in active CS $(5,16)$, and altered bone area has also been found in other endocrine disorders (37-39). While we are not aware of any earlier reports showing that successful cure of CS affects bone area, treatment with parathyroid hormone was recently shown to increase vertebral cross-sectional area in postmenopausal women with GC-induced osteoporosis (40). The anabolic effect of parathyroid hormone in their study was explained by increased bone turnover and in particular bone formation, similar to the changes in bone turnover markers observed in our study after correction of excess cortisol levels. However, it is important to consider that investigations by DXA are limited by its twodimensional acquisition plane (of a 3 dimensional structure) for determining structure and geometric parameters, and that a threshold effect on edge determination due to changes in body composition can not be ruled out. Moreover, in relation to BMD measurements and Z-scores patients with CS are especially prone to vertebral fractures, most often manifested clinically as back pain or loss of height due to compression of the vertebrae, DXA measurements may record inappropriately high BMD values in the spine.

While not all the patients fulfilled strict criteria for cure according to the low dose DST, all patients in the present study were without clinical symptoms of active disease at follow-up, with normal diurnal variation in serum cortisol levels and normal urinary excretion of free cortisol. Moreover, biochemical parameters (Table 1) and bone mass indices (BMD, BMC, area) showed no differences between patients with DST $<40 \mathrm{nmol} / \mathrm{l}$ as compared to those with DST $>40 \mathrm{nmol} / \mathrm{l}$. Interestingly however, indices of bone mass and turn-over were not correlated to cortisol levels at baseline, whereas negative correlations were demonstrated after treatment for various indices of bone mass and osteocalcin. The limited size of the study population did not allow for stratification of eugonadal and hypogonadal patients. However, we did not find evidence for a high bone-turnover state in CS patients as seen in patients with postmenopausal osteoporosis caused by estrogen deficiency. On the contrary, we observed a low bone turnover state and indications of un-coupling of the remodeling process in patients with active CS. Thus, the principal mediator of bone loss in active CS is likely to be excess GC and not hypogonadism. This is in accordance with observations in an experimental mouse model in which 

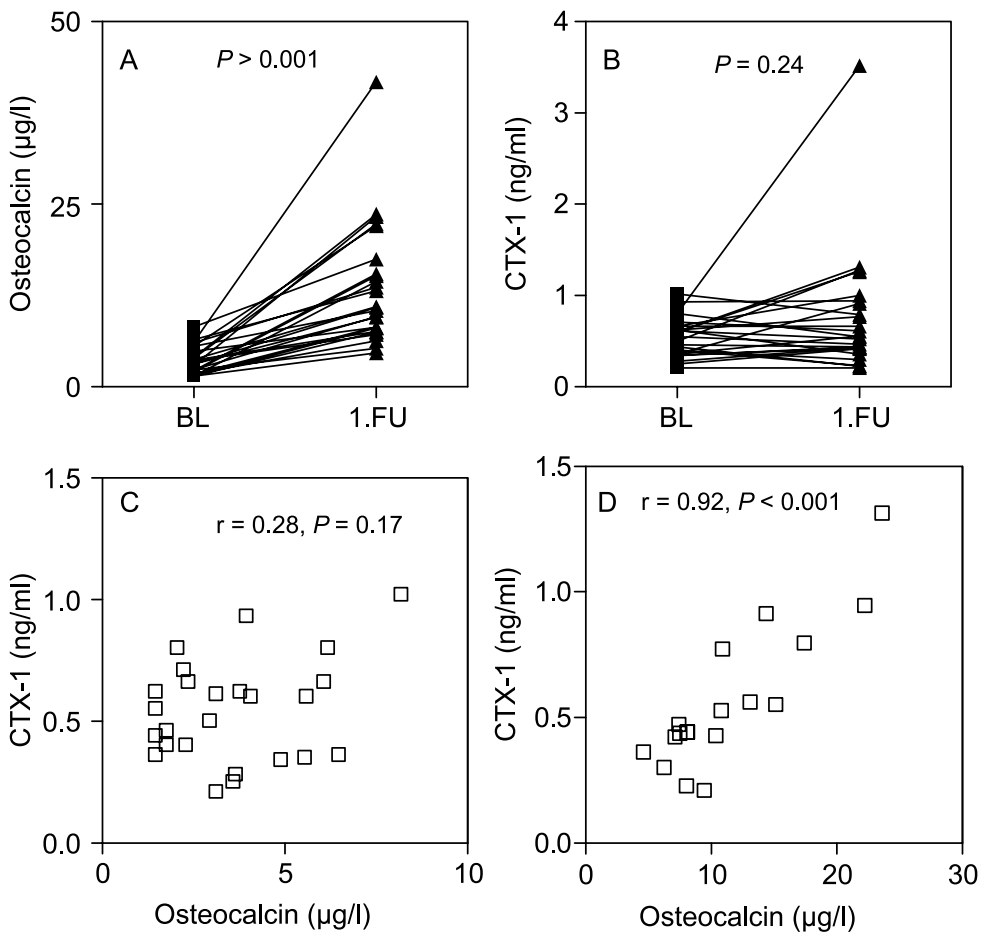

Figure 4 Longitudinal changes in serum levels of (A) osteocalcin and (B) CTX-1 at baseline (BL) and first follow-up (1.FU) after surgical treatment in CS patients $(n=25)$. Correlations $(r)$ between serum levels of osteocalcin and CTX-1 (C) before and (D) after surgical treatment, respectively. Data are given as individual observations.

excess GC seems to override the effect of sex-steroids on the skeleton (20). Moreover, we did not find evidence for anabolic skeletal effects of the androgen DHEAS in our CS population, as was suggested by a recent study in adrenal vs. pituitary CS (8), because DHEAS levels did not correlate (data not shown) with measured bone mass parameters in our study. However, these results must be interpreted with caution as we were not able to match controls and patients according to gonadal status.

Our data further suggest that the observed bone loss in active CS is due to uncoupled bone remodeling and suppressed bone formation. This is in agreement with previous reports that excess GCs cause reduction in bone formation and, to a lesser degree, increased bone resorption, implying a state of low bone turnover $(2-4,9,16,18,22,23,25)$. While the importance of suppressed bone formation secondary to reduced osteoblast number and/or activity is considered to be a central component in GC-induced bone loss, reports on effects on bone resorption have been less consistent, as there is evidence for increased $(3,9)$, decreased $(4$, 23), or unchanged (11) bone resorption. The apparent controversy as to the importance of increased bone resorption as an important component of GC-induced bone loss may in part be related to the study population; in contrast to hospitalized patients starting up GC therapy, patients with CS are likely to have manifested disease long (several years) before diagnosis and treatment. Hence, the initial resorptive phase may easily have been missed in the large majority of CS patients when they are diagnosed and selected for clinical studies. Whereas the biochemical markers of bone turnover showed no relationship at baseline, a highly significant correlation between the formative and resorptive serum markers was observed during followup, suggesting a normalization of the coupling process with the balance in favor of increased bone formation after treatment.

In conclusion, this study demonstrates generalized pronounced bone loss in active CS, not only at sites consisting mainly of trabecular bone, but also in predominantly cortical bone compartments. Biochemical markers of bone turnover indicate uncoupling of the remodeling process in active CS and association of reduced bone mass with suppressed bone formation and increased resorption. Our longitudinal data add to previous findings by showing a positive correlation between gain in BMD and follow-up time after cure at the LS and FN respectively. The accompanying changes in bone turnover markers, showing restoration of the coupling process and a marked increase in serum osteocalcin levels, indicate that enhanced bone formation is mainly responsible for improvement of bone mass after correction of excess cortisol levels. Finally, our data demonstrating successive normalization of BMD first 
at the LS, then at the FN, and finally in TB, suggest that bone loss in active CS is reversible in all measured bone compartments after prolonged recovery.

\section{References}

1 Di Somma C, Colao A, Pivonello R, Klain M, Faggiano A, Tripodi FS, Merola B, Salvatore M \& Lombardi G. Effectiveness of Chronic Treatment With Alendronate in the Osteoporosis of Cushing's Disease. Clinical Endocrinology 199848 655-662.

2 Di Somma C, Pivonello R, Loche S, Faggiano A, Marzullo P, Di Sarno A, Klain M, Salvatore M, Lombardi G \& Colao A. Severe Impairment of Bone Mass and Turnover in Cushing's Disease. Clinical Endocrinology 200256 153-158.

3 Di Somma C, Pivonello R, Loche S, Faggiano A, Klain M, Salvatore M. Lombardi G \& Colao A. Effect of 2 Years of Cortisol Normalization on the Impaired Bone Mass and Turnover in Adolescent and Adult Patients With Cushing's Disease: a Prospective Study. Clinical Endocrinology $200358302-308$.

4 Francucci CM, Pantanetti P, Garrapa GG, Massi F, Arnaldi G \& Mantero F. Bone Metabolism and Mass in Women With Cushing's Syndrome and Adrenal Incidentaloma. Clinical Endocrinology $200257587-593$.

5 Kristo C, Godang K, Ueland T, Lien E, Aukrust P, Froland SS \& Bollerslev J. Raised Serum Levels of Interleukin-8 and Interleukin-18 in Relation to Bone Metabolism in Endogenous Cushing's Syndrome. European Journal of Endocrinology 2002 146 389-395.

6 Luisetto G, Zangari M, Camozzi V, Boscaro M, Sonino N \& Fallo F. Recovery of Bone Mineral Density After Surgical Cure, but Not by Ketoconazole Treatment, in Cushing's Syndrome. Osteoporosis International 200112 956-960.

7 Manning PJ, Evans MC \& Reid IR. Normal Bone Mineral Density Following Cure of Cushing's Syndrome. Clinical Endocrinology $199236229-234$.

8 Ohmori N, Nomura K, Ohmori K, Kato Y, Itoh T \& Takano K. Osteoporosis Is More Prevalent in Adrenal Than in Pituitary Cushing's Syndrome. Endocrine Journal $2003501-7$.

9 Chiodini I, Carnevale V, Torlontano M, Fusilli S, Guglielmi G, Pileri M, Modoni S, Di Giorgio A, Liuzzi A, Minisola S, Cammisa M, Trischitta V \& Scillitani A. Alterations of Bone Turnover and Bone Mass at Different Skeletal Sites Due to Pure Glucocorticoid Excess: Study in Eumenorrheic Patients With Cushing's Syndrome. Journal of Clinical Endocrinology and Metabolism 1998 83 1863-1867.

10 Minetto M, Reimondo G, Osella G, Ventura M, Angeli A \& Terzolo M. Bone Loss Is More Severe in Primary Adrenal Than in Pituitary-Dependent Cushing's Syndrome. Osteoporosis International 200415 855-861.

11 Hermus AR, Smals AG, Swinkels LM, Huysmans DA, Pieters GF, Sweep CF, Corstens FH \& Kloppenborg PW. Bone Mineral Density and Bone Turnover Before and After Surgical Cure of Cushing's Syndrome. Journal of Clinical Endocrinology and Metabolism 1995 $802859-2865$.

12 Canalis E \& Giustina A. Glucocorticoid-Induced Osteoporosis: Summary of a Workshop. Journal of Clinical Endocrinology and Metabolism $2001865681-5685$.

13 Vestergaard P, Lindholm J, Jorgensen JO, Hagen C, Hoeck HC, Laurberg P, Rejnmark L, Brixen K, Kristensen LO, FeldtRasmussen U \& Mosekilde L. Increased Risk of Osteoporotic Fractures in Patients With Cushing's Syndrome. European Journal of Endocrinology $200214651-56$.

14 van Staa TP, Leufkens HG, Abenhaim L, Zhang B \& Cooper C. Oral Corticosteroids and Fracture Risk: Relationship to Daily and Cumulative Doses. Rheumatology 200039 1383-1389.

15 Vestergaard P, Olsen ML, Paaske Johnsen S, Rejnmark L, Sorensen HT \& Mosekilde L. Corticosteroid Use and Risk of Hip
Fracture: a Population-Based Case-Control Study in Denmark. Journal of Internal Medicine 2003254 486-493.

16 Godang K, Ueland T \& Bollerslev J. Decreased Bone Area, Bone Mineral Content, Formative Markers, and Increased Bone Resorptive Markers in Endogenous Cushing's Syndrome. European Journal of Endocrinology 1999141 126-131.

17 Karavitaki N, Ioannidis G, Giannakopoulos F, Mavrokefalos P \& Thalassinos N. Evaluation of Bone Mineral Density of the Peripheral Skeleton in Pre- and Postmenopausal Women With Newly Diagnosed Endogenous Cushing's Syndrome. Clinical Endocrinology $200460264-270$.

18 Sartorio A, Conti A, Ferrario S, Passini E, Re T \& Ambrosi B. Serum Bone Gla Protein and Carboxyterminal Cross-Linked Telopeptide of Type I Collagen in Patients With Cushing's Syndrome. Postgraduate Medical Journal 199672 419-422.

19 Boscaro M, Barzon L, Fallo F \& Sonino N. Cushing's Syndrome. Lancet $2001357783-791$.

20 Weinstein RS, Jia D, Powers CC, Stewart SA, Jilka RL, Parfitt AM \& Manolagas SC. The Skeletal Effects of Glucocorticoid Excess Override Those of Orchidectomy in Mice. Endocrinology 2004 145 1980-1987.

21 Lukert BP, Higgins JC \& Stoskopf MM. Serum Osteocalcin Is Increased in Patients With Hyperthyroidism and Decreased in Patients Receiving Glucocorticoids. Journal of Clinical Endocrinology and Metabolism $1986 \mathbf{6 2} 1056-1058$.

22 Sartorio A, Ambrosi B, Colombo P, Morabito F \& Faglia G. Osteocalcin Levels in Cushing's Disease Before and After Treatment. Hormone and Metabolic Research 19882070.

23 Cortet B, Cortet C, Blanckaert F, d'Herbomez M, Marchandise X, Wemeau J-L, Decoulx M \& Dewailly D. Quantitative Ultrasound of Bone and Markers of Bone Turnover in Cushing's Syndrome. Osteoporosis International 200112 117-123.

24 Osella G, Terzolo M, Reimondo G, Piovesan A, Pia A, Termine A, Paccotti P \& Angeli A. Serum Markers of Bone and Collagen Turnover in Patients With Cushing's Syndrome and in Subjects With Adrenal Incidentalomas. Journal of Clinical Endocrinology and Metabolism 199782 3303-3307.

25 Piovesan A, Terzolo M, Reimondo G, Pia A, Codegone A, Osella G, Boccuzzi A, Paccotti P \& Angeli A. Biochemical Markers of Bone and Collagen Turnover in Acromegaly or Cushing's Syndrome. Hormone and Metabolic Research 199426 234-237.

26 Sartorio A, Conti A, Ferrero S, Giambona S, Re T, Passini E \& Ambrosi B. Evaluation of Markers of Bone and Collagen Turnover in Patients With Active and Preclinical Cushing's Syndrome and in Patients With Adrenal Incidentaloma. European Journal of Endocrinology 1998138 146-152.

27 Takuma A, Kaneda T, Sato T, Ninomiya S, Kumegawa M \& Hakeda Y. Dexamethasone Enhances Osteoclast Formation Synergistically With Transforming Growth Factor-Beta by Stimulating the Priming of Osteoclast Progenitors for Differentiation into Osteoclasts. Journal of Biological Chemistry $2003 \mathbf{2 7 8} 44667$ 44674.

28 O'Brien CA, Jia D, Plotkin LI, Bellido T, Powers CC, Stewart SA, Manolagas SC \& Weinstein RS. Glucocorticoids Act Directly on Osteoblasts and Osteocytes to Induce Their Apoptosis and Reduce Bone Formation and Strength. Endocrinology 2004145 1835-1841.

29 Ohnaka K, Taniguchi H, Kawate H, Nawata H \& Takayanagi R. Glucocorticoid Enhances the Expression of Dickkopf-1 in Human Osteoblasts: Novel Mechanism of Glucocorticoid-Induced Osteoporosis. Biochemical and Biophysical Research Communications $2004318259-264$.

30 Weinstein RS, Jilka RL, Parfitt AM \& Manolagas SC. Inhibition of Osteoblastogenesis and Promotion of Apoptosis of Osteoblasts and Osteocytes by Glucocorticoids. Potential Mechanisms of Their Deleterious Effects on Bone. The Journal of Clinical Investigation $1998102274-282$.

31 Pereira RM, Delany AM, Durant D \& Canalis E. Cortisol Regulates the Expression of Notch in Osteoblasts. Journal of Cellular Biochemistry $2002 \mathbf{8 5} 252-258$. 
32 Eberhardt AW, Yeager-Jones A \& Blair HC. Regional Trabecular Bone Matrix Degeneration and Osteocyte Death in Femora of Glucocorticoid- Treated Rabbits. Endocrinology $2001 \mathbf{1 4 2}$ $1333-1340$.

33 Hirayama T, Sabokbar A \& Athanasou NA. Effect of Corticosteroids on Human Osteoclast Formation and Activity. Journal of Endocrinology 2002175 155-163.

34 Weinstein RS, Chen JR, Powers CC, Stewart SA, Landes RD, Bellido T, Jilka RL, Parfitt AM \& Manolagas SC. Promotion of Osteoclast Survival and Antagonism of Bisphosphonate-Induced Osteoclast Apoptosis by Glucocorticoids. The Journal of Clinical Investigation 2002109 1041-1048.

35 Arnaldi G, Angeli A, Atkinson AB, Bertagna X, Cavagnini F, Chrousos GP, Fava GA, Findling JW, Gaillard RC, Grossman AB, Kola B, Lacroix A, Mancini T, Mantero F, Newell-Price J, Nieman LK, Sonino N, Vance ML, Giustina A \& Boscaro M. Diagnosis and Complications of Cushing's Syndrome: a Consensus Statement. Journal of Clinical Endocrinology and Metabolism 2003 88 5593-5602.

36 Lunar corporation. Technical manual. Documentation version 3/95. DPX version $4.6 \mathrm{c}$.
37 Akcay MN, Akcay G \& Bilen H. The Effects of Calcitonin on Bone Resorption in Hyperthyroidism: a Placebo-Controlled Clinical Study. Journal of Bone and Mineral Metabolism 200422 90-93.

38 Chen O, Kaji H, Iu MF, Nomura R, Sowa H, Yamauchi M, Tsukamoto T, Sugimoto T \& Chihara K. Effects of an Excess and a Deficiency of Endogenous Parathyroid Hormone on Volumetric Bone Mineral Density and Bone Geometry Determined by Peripheral Quantitative Computed Tomography in Female Subjects. Journal of Clinical Endocrinology and Metabolism $2003 \mathbf{8 8} 4655-4658$.

39 Wuster C, Harle U, Rehn U, Muller C, Knauf K, Koppler D, Schwabe C \& Ziegler R. Benefits of Growth Hormone Treatment on Bone Metabolism. Bone Density and Bone Strength in Growth Hormone Deficiency and Osteoporosis. Growth Hormone \& IGF Research 19988 (Suppl A) 87-94.

40 Rehman O \& Lane NE. Effect of Glucocorticoids on Bone Density. Medical and Pediatric Oncology $200341212-216$.

Received 20 June 2005

Accepted 12 October 2005 